Original Research

\title{
Toxic and Trace Element Levels in Salmo trutta macrostigma and Oncorhynchus mykiss Trout Raised in Different Environments
}

\author{
Aslı ÇILINGİR YELTEKİN*, Emin SAĞLAMER \\ Department of Chemistry, Faculty of Science, University of Yuzuncu Yil, Van, Turkey
}

Received: 28 December 2017

Accepted: 27 April 2018

\begin{abstract}
The increase in human population along with economic and technological developments has led to significant degrees of deterioration in water quality globally. As a result, it is important to determine the toxic metal concentrations in organisms living in water, especially in commonly consumed fish species. With this aim, due to the shallow water in our region we chose the important trout production location of the Çatak River for sampling in our study, using natural Salmo trutta macrostigma and Oncorhynchus mykiss from the river and Salmo trutta macrostigma and Oncorhynchus mykiss bred in a trout production facility in Çatak District. These fish had element levels (Be, B, Al, Ti, Cr, Mn, Co, $\mathrm{Ni}, \mathrm{Cu}, \mathrm{Zn}, \mathrm{Mo}, \mathrm{Cd}, \mathrm{Ba}, \mathrm{Pb}, \mathrm{Na}, \mathrm{Mg}, \mathrm{Si}, \mathrm{K}, \mathrm{Ca}, \mathrm{Fe}, \mathrm{As}, \mathrm{Se}$, and $\mathrm{Sb}$ ) measured in heart, muscle, liver, kidney, gills, gonads, brain, and intestinal tissue using an ICP-OES device. The results of the study are grouped as toxic ( $\mathrm{Be}, \mathrm{Cd}, \mathrm{Pb}, \mathrm{Al}, \mathrm{Ba}, \mathrm{Ti})$, trace (Fe, $\mathrm{Zn}, \mathrm{Cu}, \mathrm{Mn}, \mathrm{Co}, \mathrm{Ni}, \mathrm{Cr}, \mathrm{B}, \mathrm{Si}, \mathrm{Mo}$ ) and macro (Ca, $\mathrm{Mg}, \mathrm{K}, \mathrm{Na}$ ) elements for assessment. As a result, the nutrient values of the trout grown on the farm and in the natural environment were high.
\end{abstract}

Keywords: toxic elements, trace elements, macro elements Oncorhynchus mykiss, Salmo trutta macrostigma

\section{Introduction}

Aquaculture has developed rapidly around the world. The rainbow trout (Oncorhynchus mykiss) has a place in freshwater aquaculture [1]. In this respect, rainbow trout has become a focus in terms of both economic and nutritional importance [2]. In addition to land-based food, aquaculture is important for its nutritional value. The World Health Organization has

*e-mail: aslicyeltekin@gmail.com stated that fish consumption should be $12 \mathrm{~kg}$ per year per person [3]. Today, fish are regarded as an important source of protein after investigating their components and their effect on our health [4]. Trace elements, depending on their concentration, may have beneficial or harmful effects on human health. These elements are important, as they act as antioxidants in the body, and they are the cofactors of several enzymes. They also act as the structural components of metalloenzymes and metalloproteins and are included as stabilizers in membranes [5-6].

Water resources are increasingly polluted due to rapid advances in technology and industry. Identification 
and quantification of pollution sources and protection of the quality of surface waters are crucial, since the adequate quality and quantity of water are essential for sustainable development [7]. Environmental pollution is a growing threat for the ecosystem and humans. The most dangerous environmental pollutants are heavy metals because they cannot be degraded and persist in the environment for a long time [8]. Heavy metals also have toxic effects on aquatic animals. Heavy metals form insoluble organometallic compounds in the gills of fish. These compounds are carried into the bloodstream and tissues of fish [9]. Fish are an important protein source in the biological cycle. Increased accumulation of heavy metals in fish negatively affect the health of organisms feeding on fish [10]. Heavy metals constitute a risk factor for certain diseases by impairing the stability of elements at high or low concentrations [11].

There are very few studies on trout cultivated and found naturally in Turkey. The valley of the two Çatak rivers in Van Province is important for trout production due to the cool waters. The surrounding area is forested and has a reputation for the Çatak River trout. For this reason, in this study samples were taken from the Çatak district in Van province. In the study, toxic, trace, and macro element levels in Salmo trutta macrostigma and Oncorhynchus mykiss bred on farms and living in their natural habitats were determined. These fish have great commercial significance and are consumed in large quantities by the public. The study aimed to determine the effect of heavy metals and trace elements contained in these fish on consumers' health. A comparison was also made between trace element and heavy metal levels in trout cultivated on farms and living in their natural environment.

\section{Materials and Methods}

\section{Fish Sampling}

In this study, 50 rainbow trout weighing 250-300 g aged 2-3 years were analysed. The fish

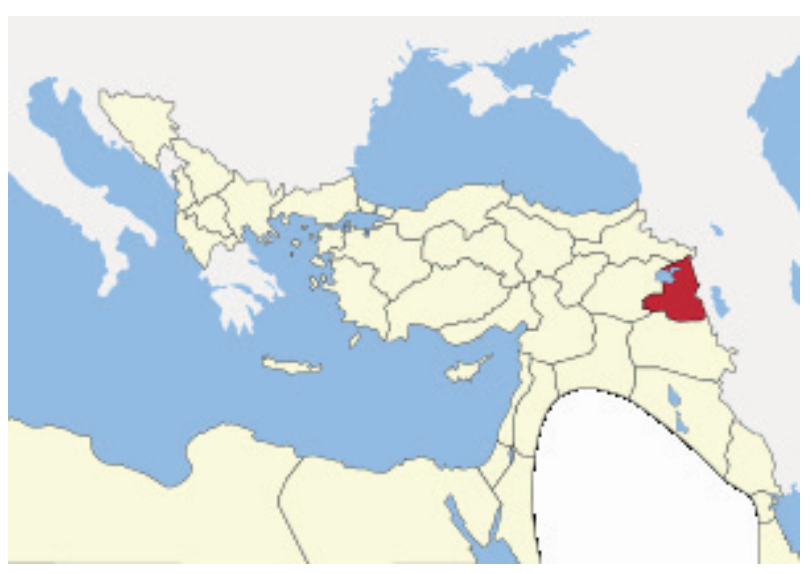

Fig. 1. Çatak township map of Van Province in Turkey. were taken from the Çatak River in Çatak District of Van Province, and from a trout farm nearby (Fig. 1). Ten freshwater trout (Salmo trutta macrostigma) and 10 rainbow trout (Oncorhynchus mykiss Walbaum 1792) were caught from the Çatak River. Fifteen freshwater trout (Salmo trutta macrostigma) and 15 rainbow trout (Oncorhynchus mykiss Walbaum 1792) were obtained from the fish farm. After the determination of weight (g) and sex under laboratory conditions, they were stored in a freezer at $-20^{\circ} \mathrm{C}$ until the analysis process of muscle, liver, gill, gonad, heart, kidney, intestinal, and brain tissues. Age determination was made based on the operculum sample taken from each fish.

\section{Sample Preparation}

Approximately $1 \mathrm{~g}$ of the tissues from the frozen fishes was weighed. The tissues, prepared according to the Alam et al. [12] method, were placed in glass tubes. Three $\mathrm{ml}$ of a $65 \%$ solution of $\mathrm{HNO}_{3}$ (Merck, Germany) and $36.5 \%$ of HCI (Merck, Germany) 1:1 solution was added to the tissues. The samples were left at room temperature for 1 hour. The tissues were kept in an incubator for 3 hours at $200^{\circ} \mathrm{C}$ for breakdown of the tissues.

$1 \mathrm{~N} 2 \mathrm{ml}$ of solution prepared with $65 \% \mathrm{HNO}_{3}$ was added to the completely dry tubes, and the drying process was repeated in the $200^{\circ} \mathrm{C}$ incubator. This process should be repeated until there is no remnant left in the tubes. In the last stage, after the dried tubes were cooled, $2.5 \mathrm{ml}$ of $1 \mathrm{~N} \mathrm{HNO}_{3}$ was added and the volume was completed to $10 \mathrm{ml}$ with deionized distilled water and prepared for analysis. Prepared tissues were analysed with ICP-OES (Thermoscientific ICAP 6000 Series) (0.005 ppm detectable limit) and the levels of $\mathrm{Be}, \mathrm{B}, \mathrm{Al}, \mathrm{Ti}, \mathrm{Cr}, \mathrm{Mn}, \mathrm{Co}, \mathrm{Ni}, \mathrm{Cu}, \mathrm{Zn}, \mathrm{Mo}, \mathrm{Cd}, \mathrm{Ba}, \mathrm{Pb}$, $\mathrm{Na}, \mathrm{Mg}, \mathrm{Si}, \mathrm{K}, \mathrm{Ca}, \mathrm{Fe}, \mathrm{As}, \mathrm{Se}$, and $\mathrm{Sb}$ elements were determined.

\section{Statistical Analysis}

Salmo trutta macrostigma and Oncorhynchus mykiss trout grown in the farm are among themselves; the Salmo trutta macrostigma and Oncorhynchus mykiss trout that grow in the natural environment are also compared among themselves. After analysis of metal levels in samples from trout species, confidence intervals were calculated by determining the standard deviations of the results obtained. One-way analysis of variance (one-way ANOVA) and Tukey tests were applied to the data, and the differences between the data were determined $(p<0.05)$.

\section{Result and Discussion}

In recent years, the amount of fish consumed globally has rapidly increased due to high-quality protein, high amounts fatty acids, and mineral content 


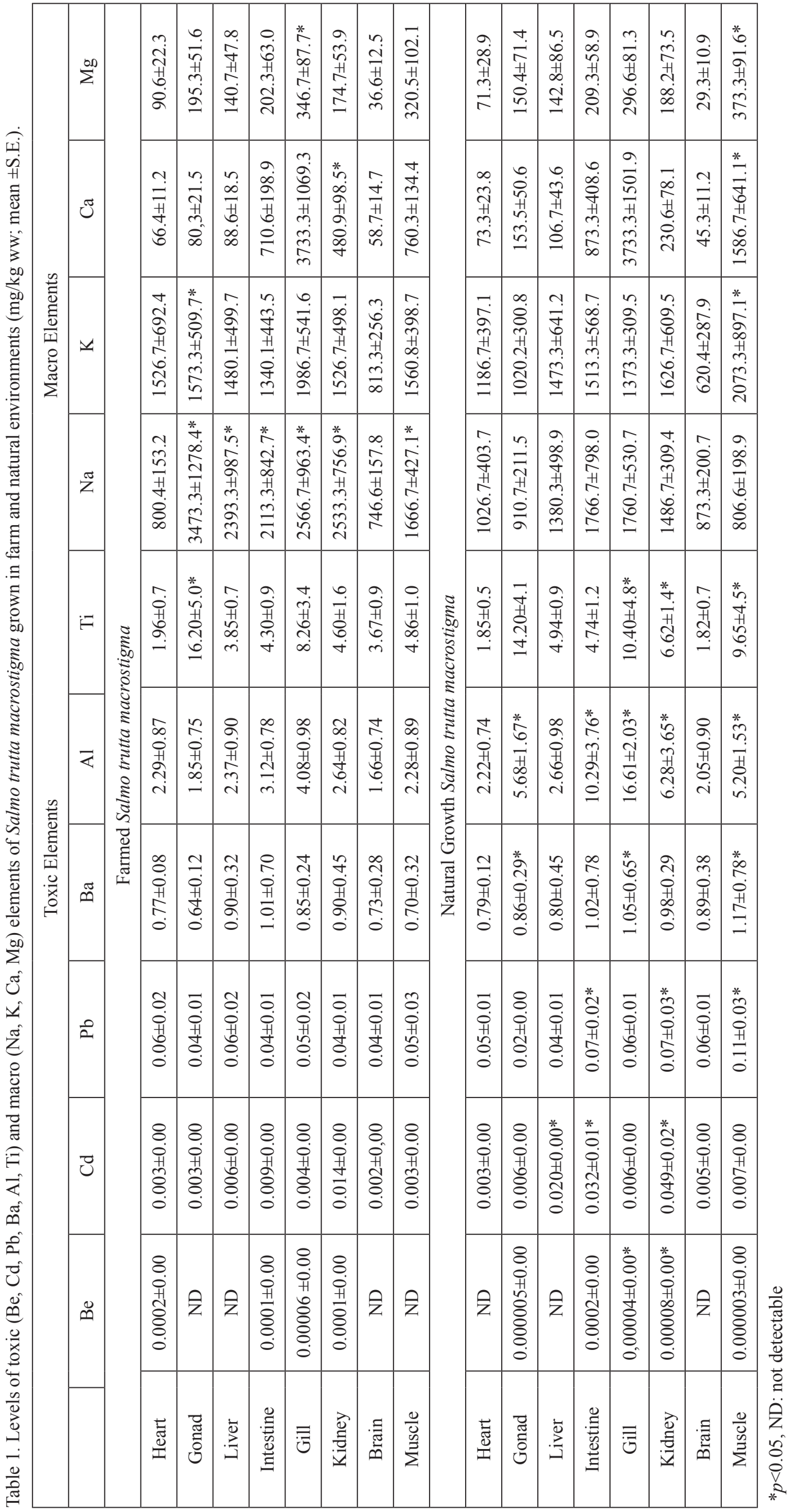




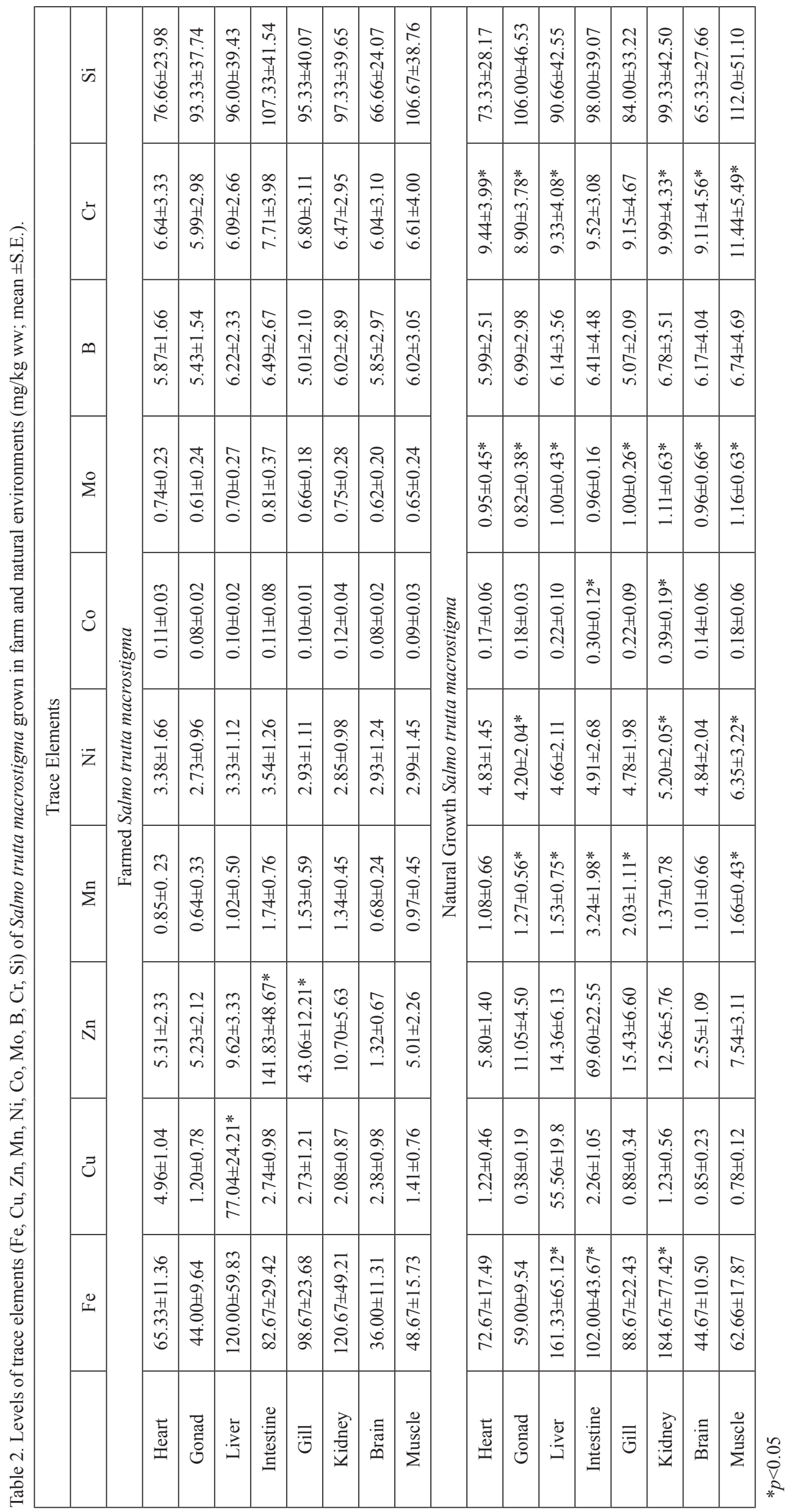


[13-14]. The increase in human population and economic and technological developments has led to significant degrees of deterioration in water quality globally [15-16]. In this situation, more and more polluting material is emptied into water resources and rivers, causing water pollution and eutrophication. As a result, it is important to determine the toxic metal concentrations in organisms living in water - especially in commonly consumed fish species [17-18].

In Salmon trutta macrostigma fish cultivated on a farm we found $\mathrm{Cr}>\mathrm{Mo}$ in the heart tissue; $\mathrm{Na}>\mathrm{K}$ $>\mathrm{Ti}>\mathrm{Cr}>\mathrm{Ni}>\mathrm{Mn}>\mathrm{Ba}>\mathrm{Mo}$ in the gonad tissues; $\mathrm{Na}>\mathrm{Fe}>\mathrm{Cu}>\mathrm{Cr}>\mathrm{Mn}>\mathrm{Mo}>\mathrm{Cd}$ elements in the liver tissue; $\mathrm{Na}>\mathrm{Fe}>\mathrm{Zn}>\mathrm{Al}>\mathrm{Mn}>\mathrm{Pb}>\mathrm{Co}>\mathrm{Cd}$ in the intestine; $\mathrm{Zn}>\mathrm{Al}>\mathrm{Ti}>\mathrm{Mn}>\mathrm{Ba}>\mathrm{Mo}>\mathrm{Be}$ in the gill tissue; $\mathrm{Na}>\mathrm{Ca}>\mathrm{Fe}>\mathrm{Cr}>\mathrm{Ti}>\mathrm{Al}>\mathrm{Ni}>$ $\mathrm{Mo}>\mathrm{Co}>\mathrm{Cd}>\mathrm{Be}$ in kidney tissue; $\mathrm{Cr}>\mathrm{Mo}$ in brain tissue; and $\mathrm{K}>\mathrm{Na}>\mathrm{Ca},>\mathrm{Mg}>\mathrm{Cr}>\mathrm{Ti}>\mathrm{Ni}>\mathrm{Al}>$ $\mathrm{Mo}>\mathrm{Ba}>\mathrm{Mn}>\mathrm{Pb}>\mathrm{Cd}$ in muscle tissues were found to be significant compared to those found in the natural environment (Tables 1-2; $p<0.05$ ).

A study of tissues from $C$. carassius and $C$. gigas fish found in Meilian Bay of Lake Taihu in China identified generally high doses of heavy metals $(\mathrm{Pb}$, $\mathrm{Cd}, \mathrm{Cr}$, and $\mathrm{Cu}$ ). The study stated that this was due to disruption of the ecosystem, industrialization, and multiple aspects of human activity [18]. Again, in the South China Sea, element levels $(\mathrm{Cd}, \mathrm{Pb}, \mathrm{Cr}, \mathrm{Ni}, \mathrm{Cu}$, and $\mathrm{Zn}$ ) were determined in naturally cultivated fish species with trade value (Thunnus obesus, Decapterus lajang, Cubiceps squamiceps, and Priacanthus macracanthus). The values found were reported not to form a danger in terms of health [19]. Varol et al. [20] researched the $\mathrm{Co}, \mathrm{Cr}, \mathrm{Cu}, \mathrm{Fe}, \mathrm{Mn}, \mathrm{Ni}, \mathrm{As}, \mathrm{Pb}, \mathrm{Cd}$, and $\mathrm{Zn}$ element levels and accumulation in the body with continuous consumption in a trace element study of rainbow trout found in a fish farm on the Euphrates River. They stated that heavy metal levels exceeded levels permitted by international health organisations. In our study, some of the values found appear to be in parallel with this study.

In Oncorhynchus mykiss fish cultivated on a farm, $\mathrm{K}>\mathrm{Cr}>\mathrm{Ni}>\mathrm{Co}>\mathrm{Pb}>\mathrm{Cd}$ in the heart; $\mathrm{K}>\mathrm{Na}>$ $\mathrm{Mg}>\mathrm{Ni}>\mathrm{Ti}$ in the gonad; $\mathrm{Na}>\mathrm{Cu}>\mathrm{Ti}>\mathrm{Mo}>\mathrm{Co}>$ $\mathrm{Pb}>\mathrm{Cd}$ in liver tissue; $\mathrm{Zn}>\mathrm{Cu}>\mathrm{Al}>\mathrm{Mo}>\mathrm{Co}>\mathrm{Pb}$ $>\mathrm{Cd}$ in the intestinal tissue; $\mathrm{Ca}>\mathrm{K}>\mathrm{Na}>\mathrm{Mg}>\mathrm{Fe}$ $>\mathrm{Ti}>\mathrm{Al}>\mathrm{Cr}>\mathrm{Zn}>\mathrm{Mn}>\mathrm{Ni}>\mathrm{Ba}>\mathrm{Co}>\mathrm{Pb}$ in the gill tissue; $\mathrm{Na}>\mathrm{Fe}>\mathrm{Cr}>\mathrm{Ti}>\mathrm{Ni}>\mathrm{Co}>\mathrm{Pb}>\mathrm{Cd}$ in kidney tissue; $\mathrm{Mg}>\mathrm{Ca}>\mathrm{Si}>\mathrm{Fe}>\mathrm{Ti}>\mathrm{Zn}>\mathrm{Al}>\mathrm{Co}$ $>\mathrm{Cd}$ in the muscle tissues were found to be significant compared to those found in the natural environment (Table 3) (Table 4; $p<0.05$ ).

A study of $M$. galloprovincialis and T. Decussatus species found in Homa Lagoon researched the $\mathrm{Hg}$, $\mathrm{Cd}, \mathrm{Pb}, \mathrm{Cr}, \mathrm{Zn}$, and $\mathrm{Cu}$ element concentrations. The study stated that heavy metal levels posed a potential risk due to increasing environmental pollution [21]. A study researching the variation of some metal levels in Alburnus tarichi fish species analyzed the presence of
$\mathrm{Be}, \mathrm{Bi}, \mathrm{Pb}, \mathrm{Cd}, \mathrm{Fe}, \mathrm{Cu}, \mathrm{Zn}, \mathrm{Se}, \mathrm{Ni}$, and $\mathrm{Mn}$ elements, They reported that this situation was a result of environmental pollution [22]. Qin et al. [23] determined the trace element levels ( $\mathrm{Li}, \mathrm{V}, \mathrm{Cr}, \mathrm{Mn}, \mathrm{Fe}, \mathrm{Ni}, \mathrm{Cu}$, Mo, Zn, Se, Sr, Al, Ti, As, Cd, Sb, Ba, Hg, Pb, and U, $\mathrm{Co}, \mathrm{Be}, \mathrm{Ga}, \mathrm{Ag}, \mathrm{Sn}, \mathrm{Te}, \mathrm{Tm}$, and $\mathrm{Tl}$ ) in carp obtained from three separate fish farms in China. In our study, the toxic element levels appear to be higher than in this study. However, the $\mathrm{Pb}$ and $\mathrm{Cd}$ toxic element levels in fish tissue were identified to be below the maximum limits determined by the Turkish Food Codex [24] and European Communities Commission Regulation [25]. In both fish species, Be element was determined in some tissues (Tables 1-3). However, the level of beryllium, which has toxic effects in fish, has not been reported in the literature. Gill tissue from the fish found in the natural environment appeared to have values higher than the previously mentioned study, especially for $\mathrm{Al}$ and $\mathrm{Ti}$ levels. This situation may be due to water pollution and agricultural pesticides used in the area surrounding the river. The levels of the toxic element $\mathrm{Ba}$ were in parallel with other literature results.

A study by Bhouri et al. [26] in Tunisia determined the $\mathrm{K}, \mathrm{Na}, \mathrm{Ca}, \mathrm{Mg}, \mathrm{Fe}, \mathrm{Zn}, \mathrm{Cu}$, and $\mathrm{Mn}$ element concentrations in muscle and liver tissue of natural sea bass and sea bass cultivated in cages at sea. The cageraised fish had higher $\mathrm{Mn}$ and $\mathrm{Zn}$ element concentrations compared to the fish found in the natural environment. They stated that the difference in these values may be due to feeding. In our study, element levels in fish species found in both environments were in parallel with these study results.

Avigliano et al. [27] researched the heavy metal and trace element levels in muscle tissue of silverside fish found in different regions in Argentina. The study identified As, Ag, B, Ba, Bi, Ca, Cd, Co, Cr, Fe, Ga, $\mathrm{Hg}, \mathrm{K}, \mathrm{Li}, \mathrm{Mg}, \mathrm{Mn}, \mathrm{Mo}, \mathrm{Na}, \mathrm{Ni}, \mathrm{Pb}, \mathrm{Rb}, \mathrm{Sb}, \mathrm{Se}, \mathrm{Sn}, \mathrm{Sr}$, $\mathrm{Te}, \mathrm{Ti}, \mathrm{U}, \mathrm{V}$, and $\mathrm{Zn}$ element levels. They found toxic $\mathrm{As}, \mathrm{Hg}$, and $\mathrm{Pb}$ levels high enough to threaten health. In our study, the Salmo trutta macrostigma found in the natural environment had higher $\mathrm{Cd}, \mathrm{Pb}, \mathrm{Ba}$, $\mathrm{Al}, \mathrm{Fe}, \mathrm{Mn}, \mathrm{Ni}, \mathrm{Co}$, and $\mathrm{Cr}$ elements, while the farmcultivated fish had higher $\mathrm{Na}, \mathrm{Cu}$, and $\mathrm{Zn}$ elements in some tissues, with significant differences (Tables 1-2). For the Oncorhynchus mykiss species, fish in the natural environment had higher levels of $\mathrm{Cd}, \mathrm{Pb}, \mathrm{Ba}$, $\mathrm{Al}, \mathrm{Fe}, \mathrm{Mn}, \mathrm{Ni}$, and $\mathrm{Cr}$, while farm fish had higher Mo with significant differences (Tables 3-4). These differences are considered to be due to fish on farms being fed feed in a springwater environment, while fish in the natural environment feed on aquatic organisms in the river affected by all sorts of external factors. A study was carried out to evaluate the trace element bioaccumulation of the Krka River to assess the present pollution status and the analysis of various biomarkers in the gill and cytoplasm (Salmo trutta L.) of brown trout. Concentrations of trace elements in the gill and cytosol were determined by ICP-MS. Seven (Co, Cu, $\mathrm{Fe}, \mathrm{Mn}, \mathrm{Se}, \mathrm{V}$, and $\mathrm{Zn}$ ) and six (As, Cd, Cs, Rb, Sr, 


\begin{tabular}{|c|c|c|c|c|c|c|c|c|c|c|c|c|c|c|c|c|c|c|c|}
\hline \multirow{4}{*}{ 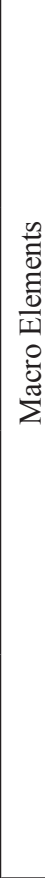 } & $\sum^{50}$ & & 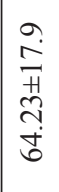 & 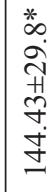 & $\begin{array}{l}0 \\
\infty \\
0 \\
0 \\
m \\
m \\
\stackrel{2}{N} \\
\stackrel{\sim}{N}\end{array}$ & 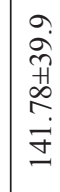 & 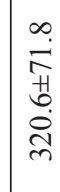 & 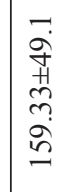 & 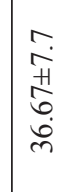 & 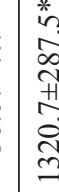 & & 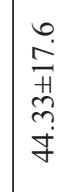 & 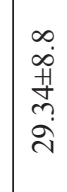 & 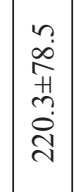 & 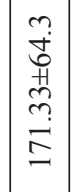 & 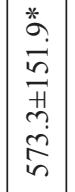 & $\left|\begin{array}{c}\vec{j} \\
\dot{\vec{d}} \\
\dot{H} \\
m \\
\tilde{n} \\
\vec{v}\end{array}\right|$ & 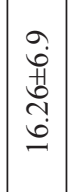 & 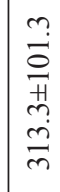 \\
\hline & ש & & $\begin{array}{l}\hat{a} \\
\frac{1}{H} \\
\hat{a} \\
\dot{\sigma} \\
+\end{array}$ & 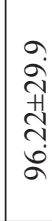 & 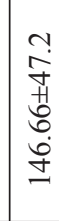 & 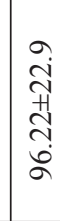 & 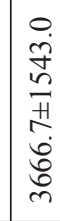 & $\begin{array}{l}0 \\
0 \\
\dot{0} \\
0 \\
0 \\
0 \\
0 \\
0 \\
\sim\end{array}$ & 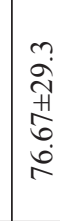 & $\begin{array}{l}* \\
0 \\
0 \\
0 \\
0 \\
0 \\
0 \\
2 \\
\alpha \\
\alpha\end{array}$ & & 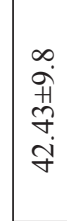 & 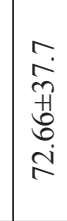 & $\begin{array}{c}\hat{\tilde{D}} \\
\dot{0} \\
\tilde{n} \\
\tilde{\sigma} \\
\tilde{2}\end{array}$ & 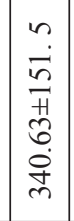 & 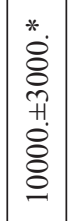 & $\left|\begin{array}{l}n \\
o \\
0 \\
0 \\
m \\
m \\
\tilde{m} \\
\tilde{m}\end{array}\right|$ & 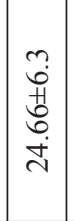 & 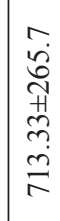 \\
\hline & $\forall$ & & 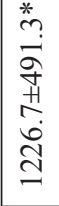 & 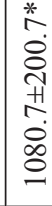 & 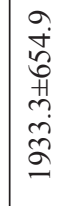 & 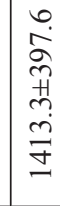 & 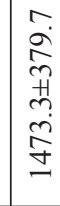 & $\begin{array}{l}0 \\
0 \\
0 \\
0 \\
0 \\
0 \\
0 \\
0 \\
n \\
n\end{array}$ & 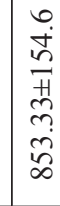 & $\mid \begin{array}{l}\bar{\alpha} \\
0 \\
0 \\
+ \\
0 \\
\delta \\
0\end{array}$ & & 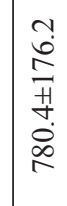 & $\begin{array}{l}\infty \\
\text { in } \\
i \\
\vec{m} \\
\tilde{n} \\
i \\
i n\end{array}$ & $\begin{array}{c}0 \\
0 \\
0 \\
0 \\
+1 \\
\hat{0} \\
0 \\
\Xi \\
=\end{array}$ & 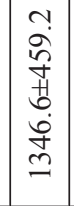 & $\left|\begin{array}{c}* \\
+ \\
\infty \\
o \\
o \\
+1 \\
\dot{0} \\
0 \\
0\end{array}\right|$ & 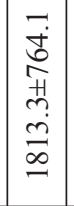 & 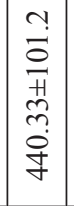 & 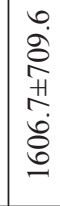 \\
\hline & $\tilde{z}$ & & 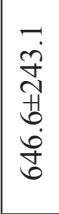 & 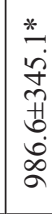 & 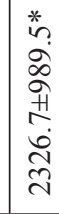 & 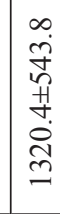 & 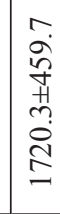 & 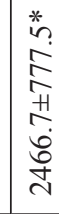 & 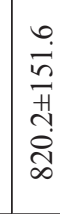 & $\begin{array}{l}0 \\
0 \\
0 \\
0 \\
0 \\
0 \\
\vdots \\
0\end{array}$ & & \begin{tabular}{l}
$n$ \\
$\infty$ \\
$\infty$ \\
$\tilde{H}$ \\
$\tilde{n}$ \\
$\tilde{2}$ \\
\multirow{f}{*}{}
\end{tabular} & 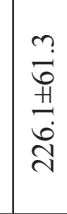 & 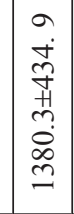 & 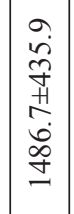 & 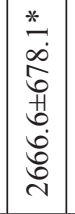 & 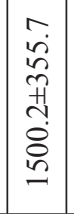 & 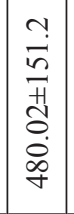 & $\begin{array}{l}r \\
\tilde{\delta} \\
0 \\
0 \\
0 \\
6 \\
\dot{0} \\
\infty \\
\infty\end{array}$ \\
\hline \multirow{7}{*}{ 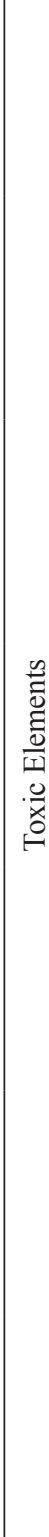 } & $F$ & 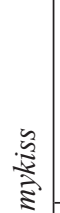 & $\begin{array}{l}2 \\
\dot{0} \\
0 \\
\stackrel{+1}{0} \\
\stackrel{-}{-}\end{array}$ & $\begin{array}{l}* \\
n \\
n \\
H \\
\simeq \\
\simeq \\
\simeq\end{array}$ & $\begin{array}{l}\text { to } \\
\text { in } \\
\text { Hै } \\
\text { nn } \\
\text { f }\end{array}$ & 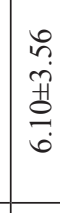 & 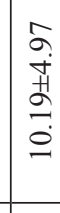 & $\begin{array}{l}\text { t } \\
\text { iv } \\
\text { tै } \\
\stackrel{f}{+}\end{array}$ & $\begin{array}{l}\hat{\infty} \\
0 \\
\text { 草 } \\
\text { r. }\end{array}$ & $\frac{\sigma}{\tilde{H}}$ & 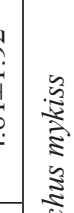 & 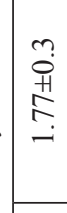 & 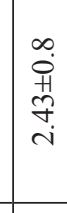 & 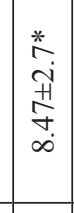 & 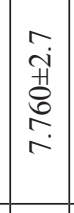 & 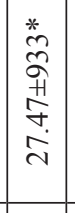 & $\mid \begin{array}{c}* \\
\tilde{n} \\
\tilde{n} \\
\tilde{H} \\
0 \\
\infty \\
\infty\end{array}$ & 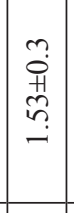 & $\begin{array}{l}* \\
\infty \\
i \\
i \\
0 \\
\infty \\
\infty \\
\infty\end{array}$ \\
\hline & « & 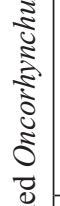 & 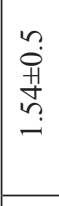 & 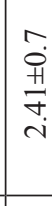 & 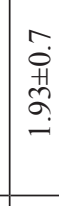 & $\begin{array}{l}\text { ? } \\
\text { Oे } \\
\text { के } \\
\text { - }\end{array}$ & $\begin{array}{l}\hat{0} \\
\hat{H} \\
\hat{b} \\
\text { in }\end{array}$ & 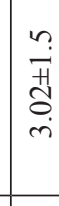 & $\begin{array}{l}0 \\
0 \\
\text { in } \\
? \\
?\end{array}$ & 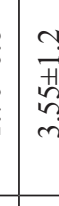 & 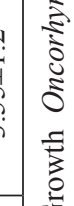 & $\begin{array}{l}\vec{i} \\
\vec{i} \\
\overrightarrow{2} \\
i \\
\dot{m}\end{array}$ & 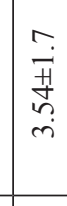 & 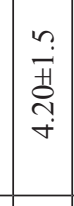 & $\mid \begin{array}{c}* \\
2 \\
n \\
n \\
\infty \\
\infty \\
n \\
a\end{array}$ & $\mid \begin{array}{l}* \\
0 \\
\infty \\
0 \\
1 \\
0 \\
0 \\
0 \\
0\end{array}$ & $\mid \begin{array}{c}r \\
\dot{\vec{H}} \\
0 \\
\dot{0} \\
\dot{r}\end{array}$ & $\begin{array}{l}\hat{0} \\
\dot{1} \\
\alpha \\
\dot{-}\end{array}$ & 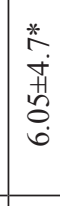 \\
\hline & $\ddot{\oplus}$ & 践 & $\begin{array}{l}\overrightarrow{0} \\
\text { 贲 } \\
\text { O. }\end{array}$ & 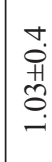 & 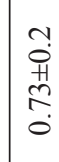 & $\begin{array}{l}n \\
0 \\
0 \\
0 \\
0 \\
0 \\
0\end{array}$ & 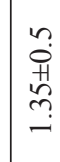 & $\begin{array}{l}\hat{0} \\
\hat{+} \\
\hat{\sigma}\end{array}$ & $\begin{array}{l}n \\
0 \\
1 \\
? \\
0 \\
0\end{array}$ & $\mid \begin{array}{l}0 \\
0 \\
0 \\
0 \\
0 \\
0\end{array}$ & 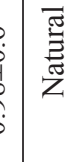 & $\begin{array}{l}+ \\
0 \\
0 \\
0 \\
0 \\
0 \\
0\end{array}$ & $\begin{array}{l}\hat{0} \\
\dot{1} \\
\alpha \\
0 \\
0 \\
0\end{array}$ & 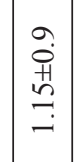 & $\left|\begin{array}{c}\infty \\
0 \\
0 \\
+1 \\
\\
-1\end{array}\right|$ & 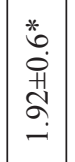 & $\left|\begin{array}{c}+ \\
0 \\
0 \\
0 \\
0 \\
0\end{array}\right|$ & $\left|\begin{array}{c}m \\
0 \\
1 \\
\hat{\imath} \\
0\end{array}\right|$ & 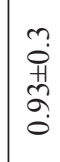 \\
\hline & 2 & & 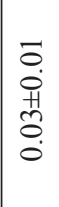 & $\begin{array}{l}\overline{0} \\
0 \\
0 \\
0 \\
0 \\
0 \\
0\end{array}$ & 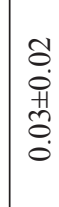 & $\begin{array}{l}\overrightarrow{0} \\
0 \\
01 \\
0 \\
0 \\
0\end{array}$ & $\begin{array}{l}\overline{0} \\
\dot{0} \\
\ddot{1} \\
0 \\
0 \\
0\end{array}$ & $\begin{array}{l}\text { ô } \\
0 \\
0 \\
\dot{y} \\
0 \\
0\end{array}$ & $\begin{array}{l}\overrightarrow{0} \\
0 \\
0 \\
+ \\
\dot{y} \\
0 \\
0\end{array}$ & $\mid \begin{array}{l}0 \\
0 \\
0 \\
0 \\
0 \\
0\end{array}$ & & \begin{tabular}{l}
$*$ \\
\multirow{2}{*}{} \\
0 \\
0 \\
0 \\
0 \\
0 \\
0
\end{tabular} & $\begin{array}{l}\overrightarrow{0} \\
0 \\
0 \\
\hat{H} \\
0 \\
0 \\
0\end{array}$ & 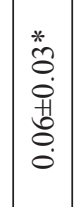 & 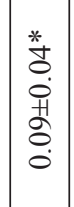 & 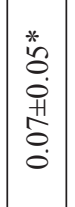 & $\left|\begin{array}{l}* \\
\tilde{o} \\
\dot{0} \\
\ddot{1} \\
0 \\
0 \\
\dot{0}\end{array}\right|$ & $\left|\begin{array}{l}0 \\
0 \\
0 \\
\dot{H} \\
0 \\
0 \\
0\end{array}\right|$ & $\begin{array}{l}\overrightarrow{0} \\
\dot{0} \\
\text { +1 } \\
\dot{0} \\
\dot{0}\end{array}$ \\
\hline & $\tilde{U}$ & & \begin{tabular}{l}
8 \\
8 \\
0 \\
+ \\
\multirow{H}{*}{} \\
8 \\
0 \\
0
\end{tabular} & 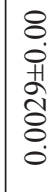 & $\begin{array}{l}8 \\
0 \\
\text { H } \\
\text { I } \\
0 \\
0\end{array}$ & 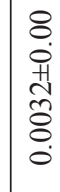 & $\begin{array}{l}8 \\
8 \\
0 \\
0 \\
y \\
y \\
0 \\
0\end{array}$ & $\begin{array}{c}8 \\
0 \\
\text { ते } \\
\text { مे } \\
0 \\
0 \\
0\end{array}$ & 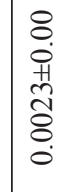 & $\mid \begin{array}{l}8 \\
o \\
0 \\
y \\
\tilde{z} \\
0 \\
0\end{array}$ & & 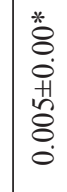 & $\begin{array}{l}8 \\
0 \\
0 \\
+1 \\
\text { f } \\
8 \\
0 \\
0\end{array}$ & 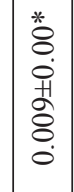 & 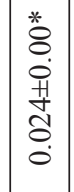 & $\left|\begin{array}{l}8 \\
0 \\
0 \\
01 \\
8 \\
0 \\
0\end{array}\right|$ & 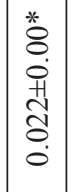 & $\left|\begin{array}{l}8 \\
0 \\
0 \\
01 \\
0 \\
\tilde{8} \\
\dot{0}\end{array}\right|$ & 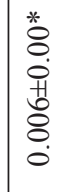 \\
\hline & $\ddot{\oplus}$ & & そ) & 之 & 学 & 吾 & 之 & 之 & 乙 & Z & & 合 & 之 & 之 & 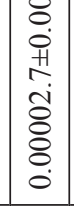 & 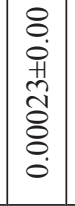 & 至 & 至 & 之 \\
\hline & & & 苛 & 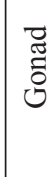 & 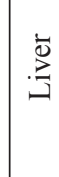 & 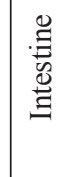 & 宁 & 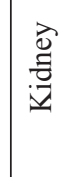 & 墕 & $\begin{array}{l}\frac{0}{0} \\
\bar{g} \\
\Sigma\end{array}$ & & $\underset{\mathbb{E}}{\mathbb{E}}$ & $\begin{array}{l}\overrightarrow{\tilde{J}} \\
\overline{\tilde{J}} \\
0\end{array}$ & $\stackrel{\vec{D}}{.}$ & 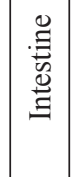 & 宁 & 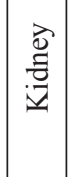 & 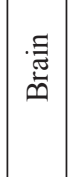 & $\begin{array}{l}\frac{0}{0} \\
0 \\
D^{2}\end{array}$ \\
\hline
\end{tabular}




\begin{tabular}{|c|c|c|c|c|c|c|c|c|c|c|c|c|c|c|c|c|c|c|c|}
\hline & $\ddot{n}$ & & 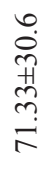 & $\begin{array}{l}\infty \\
\dot{n} \\
+ \\
+ \\
m \\
\stackrel{0}{0}\end{array}$ & 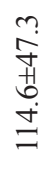 & 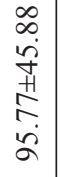 & 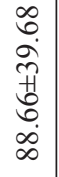 & 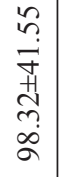 & 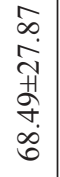 & 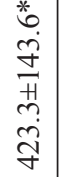 & & 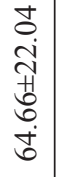 & $\begin{array}{l}\stackrel{\partial}{\tilde{y}} \\
f \\
\dot{H} \\
\dot{0} \\
\dot{0}\end{array}$ & 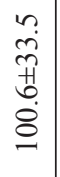 & $\begin{array}{l}n \\
\infty \\
0 \\
+ \\
\infty \\
0 \\
\ddots\end{array}$ & $\begin{array}{l}0 \\
\stackrel{r}{f} \\
\text { 苦 } \\
\dot{ \pm} \\
=\end{array}$ & $\begin{array}{l}\hat{i} \\
\tilde{n} \\
+1 \\
\dot{0} \\
\stackrel{0}{0}\end{array}$ & $\begin{array}{l}\text { i. } \\
\vec{T} \\
\vec{t} \\
\text { o } \\
\text { i }\end{array}$ & $\begin{array}{l}\vec{m} \\
\tilde{f} \\
+ \\
m \\
\ddot{\Xi} \\
=\end{array}$ \\
\hline & ש & & 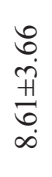 & 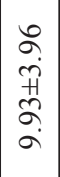 & 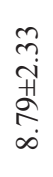 & 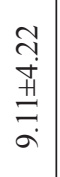 & 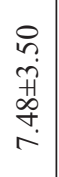 & 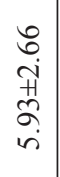 & 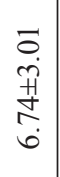 & $\begin{array}{l}\infty \\
\stackrel{\infty}{+} \\
\dot{+} \\
\underset{\sigma}{ } \\
\infty\end{array}$ & & 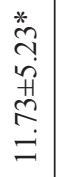 & $\begin{array}{l}\stackrel{2}{ } \\
\tilde{m} \\
\ddot{2} \\
\stackrel{2}{a}\end{array}$ & $\begin{array}{c}m \\
\tilde{n} \\
\stackrel{H}{2} \\
\sigma \\
\sigma\end{array}$ & 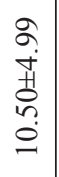 & 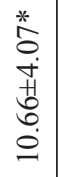 & \begin{tabular}{l}
$*$ \\
\multirow{0}{0}{} \\
$\dot{H}$ \\
+ \\
$\infty$ \\
$\infty$ \\
$a$
\end{tabular} & $\begin{array}{l}* \\
\infty \\
\infty \\
m \\
\tilde{n} \\
m \\
a\end{array}$ & 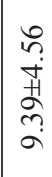 \\
\hline & $\infty$ & & 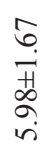 & $\begin{array}{l}\tilde{M} \\
\stackrel{i}{1} \\
\stackrel{i}{N} \\
\end{array}$ & 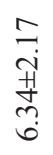 & 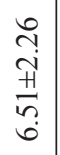 & 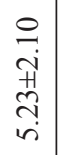 & $\begin{array}{l}\text { oे } \\
\text { in } \\
\hat{n} \\
\hat{\sigma}\end{array}$ & 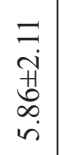 & $\begin{array}{l}\stackrel{0}{0} \\
\underset{+}{\mathbb{N}} \\
\text { }\end{array}$ & & 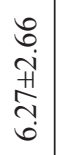 & 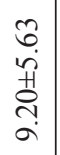 & $\begin{array}{l}\vec{f} \\
\stackrel{0}{n} \\
\stackrel{n}{r}\end{array}$ & 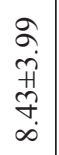 & 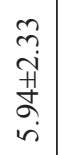 & 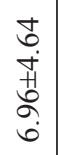 & $\begin{array}{l}\infty \\
2 \\
0 \\
\hat{n} \\
\hat{n} \\
0\end{array}$ & $\begin{array}{l}\hat{\sigma} \\
\dot{p} \\
\tilde{n} \\
\tilde{n}\end{array}$ \\
\hline & $\stackrel{0}{\Sigma}$ & & $\begin{array}{l}\text { ते } \\
\text { Oे } \\
\frac{1}{0} \\
0 \\
0\end{array}$ & $\mid \begin{array}{c}m \\
3 \\
0 \\
\vdots \\
2 \\
0 \\
0\end{array}$ & 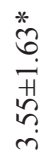 & 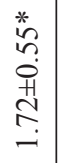 & $\begin{array}{l}\stackrel{1}{1} \\
0 \\
⿱ 1 \\
0 \\
0 \\
0\end{array}$ & $\begin{array}{l}0 \\
\tilde{0} \\
+ \\
\text { d̦ } \\
0 \\
0\end{array}$ & $\begin{array}{l}\tilde{n} \\
0 \\
0 \\
\hat{n} \\
0 \\
0\end{array}$ & 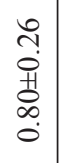 & & $\begin{array}{l}\hat{y} \\
o \\
+1 \\
\infty \\
\stackrel{-}{-}\end{array}$ & $\begin{array}{c}m \\
\stackrel{0}{0} \\
+ \\
\infty \\
\infty \\
0\end{array}$ & $\begin{array}{c}m \\
\dddot{0} \\
\stackrel{1}{1} \\
\stackrel{-}{-}\end{array}$ & 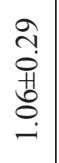 & 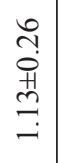 & $\begin{array}{l}0 \\
\tilde{n} \\
0 \\
0 \\
0 \\
-\end{array}$ & 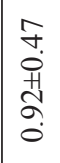 & $\begin{array}{l}m \\
\tilde{o} \\
0 \\
\tilde{n} \\
\sigma \\
0\end{array}$ \\
\hline & 8 & & $\begin{array}{l}8 \\
\stackrel{0}{0} \\
\text { In } \\
\stackrel{0}{0} \\
0\end{array}$ & $\mid \begin{array}{c}0 \\
0 \\
\dot{0} \\
\stackrel{+}{1} \\
\\
0\end{array}$ & 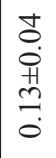 & 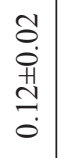 & $\begin{array}{l}\tilde{O} \\
0 \\
\stackrel{+}{H} \\
\exists \\
0\end{array}$ & 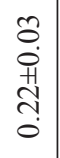 & $\begin{array}{l}0 \\
0 \\
0 \\
+1 \\
0 \\
0 \\
0\end{array}$ & 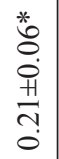 & & $\begin{array}{l}\frac{*}{0} \\
0 \\
\dot{0} \\
\ddot{1} \\
\stackrel{0}{0}\end{array}$ & 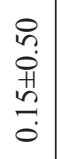 & 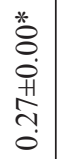 & $\begin{array}{l}\stackrel{*}{*} \\
\stackrel{0}{0} \\
+ \\
\stackrel{1}{0} \\
\stackrel{0}{0}\end{array}$ & 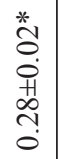 & $\begin{array}{l}\stackrel{*}{\circ} \\
\stackrel{0}{0} \\
\stackrel{+}{+} \\
\stackrel{m}{0}\end{array}$ & $\begin{array}{l}\overrightarrow{0} \\
0 \\
0 \\
m \\
0 \\
0\end{array}$ & $\begin{array}{l}\tau \\
0 \\
0 \\
0 \\
1 \\
0 \\
0 \\
0\end{array}$ \\
\hline 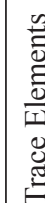 & $\bar{z}$ & & 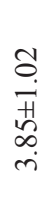 & $\begin{array}{l}\hat{\sigma} \\
\overrightarrow{\dot{H}} \\
\dot{\vec{\sigma}} \\
\dot{r}\end{array}$ & 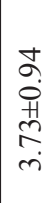 & $\begin{array}{c}\exists \\
\exists \\
\vec{H} \\
心 \\
\infty \\
\dot{r}\end{array}$ & 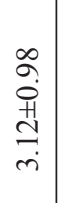 & $\begin{array}{l}n \\
\infty \\
0 \\
+ \\
\hat{n} \\
i \\
n\end{array}$ & 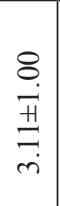 & 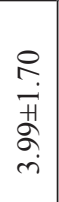 & & 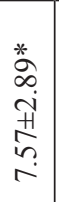 & 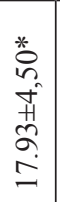 & 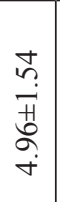 & $\begin{array}{c}\stackrel{a}{o} \\
\text { i } \\
\text { t } \\
\text { in }\end{array}$ & $\begin{array}{l}* \\
\infty \\
0 \\
i \\
+ \\
m \\
m \\
i n\end{array}$ & $\begin{array}{l}\text { * } \\
\hat{i} \\
\text { i } \\
\text { ôे } \\
\text { in }\end{array}$ & $\begin{array}{l}n \\
\sim \\
+1 \\
\infty \\
\stackrel{0}{+} \\
\dot{+}\end{array}$ & 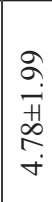 \\
\hline & $\Sigma_{\Sigma}$ & & 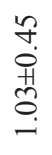 & $\mid \begin{array}{c}0 \\
m \\
0 \\
0 \\
\infty \\
0 \\
0 \\
-1\end{array}$ & 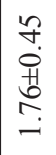 & 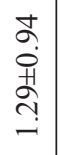 & 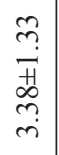 & 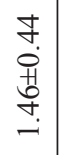 & $\begin{array}{l}m \\
\tilde{0} \\
0 \\
0 \\
\infty \\
0 \\
0\end{array}$ & $\begin{array}{c}0 \\
\stackrel{0}{0} \\
\stackrel{1}{0} \\
\stackrel{i}{i}\end{array}$ & & $\begin{array}{c}\stackrel{g}{+} \\
\stackrel{0}{+} \\
\stackrel{+}{m} \\
\end{array}$ & 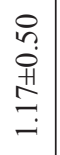 & $\begin{array}{c}\stackrel{8}{0} \\
\stackrel{0}{1} \\
\infty \\
\vec{i}\end{array}$ & $\begin{array}{c}\hat{n} \\
\dot{0} \\
+ \\
\hat{n} \\
i\end{array}$ & \begin{tabular}{l}
$*$ \\
\multirow{0}{0}{} \\
i \\
Hे \\
oे \\
in
\end{tabular} & 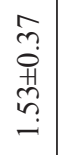 & $\begin{array}{l}0 \\
\stackrel{1}{1} \\
0 \\
\stackrel{+}{1} \\
0 \\
-1\end{array}$ & 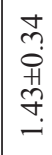 \\
\hline & ㄱ & 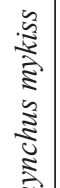 & 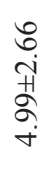 & 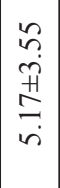 & $\begin{array}{l}\overrightarrow{0} \\
0 \\
0 \\
0 \\
0 \\
\\
=\end{array}$ & $\begin{array}{l}\stackrel{2}{2} \\
\stackrel{y}{+} \\
\stackrel{+}{r} \\
r\end{array}$ & 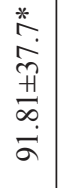 & 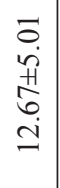 & $\begin{array}{l}\stackrel{0}{2} \\
\stackrel{0}{+} \\
\stackrel{+}{f} \\
\stackrel{-}{-}\end{array}$ & 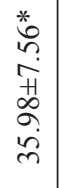 & 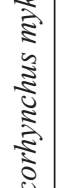 & $\begin{array}{c}8 \\
\stackrel{i}{+} \\
\stackrel{n}{n} \\
\dot{m}\end{array}$ & 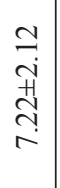 & $\begin{array}{l}\infty \\
\infty \\
i \\
\tilde{n} \\
\tilde{n} \\
\ddot{n}\end{array}$ & 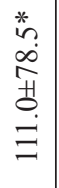 & $\begin{array}{l}\vec{n} \\
\underset{\sim}{+} \\
\forall \\
\stackrel{+}{+}\end{array}$ & 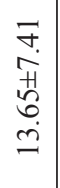 & 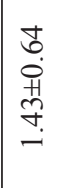 & $\begin{array}{l}\vec{\sigma} \\
\sim \\
+ \\
\infty \\
\end{array}$ \\
\hline & $\tilde{J}$ & 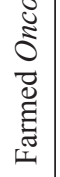 & $\begin{array}{l}0 \\
\text { ?! } \\
\text { +⿱ } \\
+ \\
\vdots \\
0\end{array}$ & $\begin{array}{l}0 \\
0 \\
0 \\
+1 \\
\text { I } \\
0\end{array}$ & $\begin{array}{l}\hat{\overrightarrow{0}} \\
\vec{H} \\
0 \\
\dot{0} \\
\dot{i}\end{array}$ & 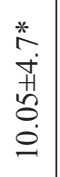 & $\begin{array}{l}\circ \\
\stackrel{0}{0} \\
+1 \\
\stackrel{0}{0} \\
0\end{array}$ & 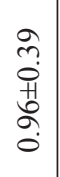 & \begin{tabular}{l} 
ते \\
0 \\
01 \\
\multirow{0}{0}{} \\
0
\end{tabular} & 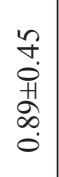 & 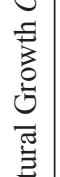 & $\begin{array}{c}m \\
\tilde{0} \\
0 \\
+ \\
\stackrel{+}{0} \\
-\end{array}$ & $\begin{array}{c}\tilde{n} \\
\tilde{o} \\
+ \\
\vdots \\
\vdots \\
0\end{array}$ & 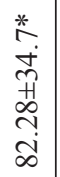 & $\begin{array}{c}\stackrel{P}{1} \\
\dot{0} \\
+ \\
\infty \\
\infty \\
-\end{array}$ & 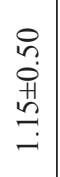 & 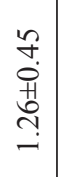 & $\begin{array}{l}2 \\
0 \\
0 \\
0 \\
0 \\
i \\
i\end{array}$ & 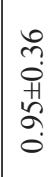 \\
\hline & 讧 & & 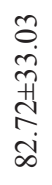 & 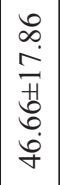 & 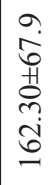 & $\begin{array}{c}\tilde{\sigma} \\
\dot{0} \\
\tilde{D} \\
\infty \\
\infty \\
\dot{0} \\
0\end{array}$ & 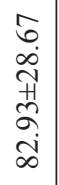 & $\begin{array}{l}0 \\
\dot{n} \\
n \\
+ \\
m \\
\stackrel{=}{=}\end{array}$ & 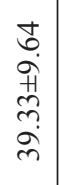 & $\begin{array}{l}\hat{m} \\
\underset{T}{1} \\
m \\
\tilde{n} \\
\stackrel{n}{2}\end{array}$ & & 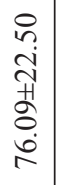 & 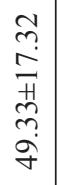 & 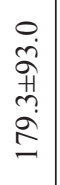 & $\begin{array}{l}0 \\
0 \\
\stackrel{0}{0} \\
\text { 1 } \\
\infty \\
0 \\
\infty \\
\infty\end{array}$ & 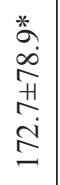 & 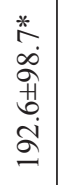 & 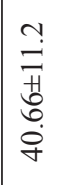 & 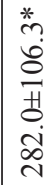 \\
\hline & & & 蒂 & $\mid \begin{array}{l}\tilde{Z} \\
\tilde{\Xi} \\
\dot{0}\end{array}$ & $\stackrel{\vec{D}}{\stackrel{\Delta}{~}}$ & 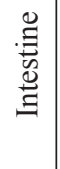 & ज्ञ & 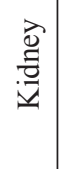 & 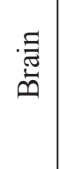 & $\begin{array}{l}\frac{0}{0} \\
\text { D } \\
\Sigma\end{array}$ & & 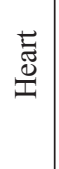 & $\begin{array}{l}\vec{J} \\
\tilde{\Xi} \\
0\end{array}$ & $\stackrel{\grave{\Delta}}{\stackrel{\Xi}{コ}}$ & 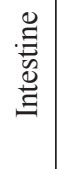 & $\overline{0}$ & 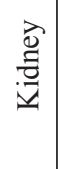 & $\begin{array}{l}\text { 寻 } \\
\text { ต }\end{array}$ & $\begin{array}{l}\frac{0}{0} \\
\underline{\mathscr{z}} \\
\sum^{2}\end{array}$ \\
\hline
\end{tabular}


and $\mathrm{Tl}$ ) elements were determined in the analysis [28]. It is seen that the results of our work are in parallel with this study.

\section{Conclusions}

In conclusion, the Çatak District and Çatak River in Van Province were chosen for sampling due to their importance for commercial trout production. Natural Salmo trutta macrostigma and Oncorhynchus mykiss in the Catak River and farm-cultivated Salmo trutta macrostigma and Oncorhynchus mykiss had toxic, trace, and macro element levels analysed in heart, muscle, liver, kidney, gill, gonad, brain, and intestinal tissues. In line with the obtained results, both fish species in the natural environment and cultivated on farms had high nutritional quality in terms of trace element and macro element levels. The dietary patterns of fish and the different water sources they live in may lead to the presence of different elemental values between the two groups. Furthermore, differences observed in element concentrations between the two fish groups are considered to vary linked to environmental conditions, mobility of fish, nutritional differences, and capacity for element accumulation. Additionally, the lack of identification of the Se element, with great importance for metabolism, and the toxic As and Sn elements is considered to be due to interaction between the elements during ICP-OES device measurements. As a result, it can be said that both of the trout grown in the farm and in the natural environment have high nutritional value.

\section{Acknowledgements}

This work was supported by the FYL-2017-6305 project of Van Yuzuncu Yil University Scientific Research Projects the Presidency. We thank the directorate of scientific research projects of Van Yuzuncu Yil University for its financial support.

\section{Conflict of Interest}

The authors declare no conflict of interest.

\section{References}

1. KIR I., TUMANTOZLU H. An Investigation of Some Heavy Metal Accumulation in Water, Sediment and Carp (Cyprinus carpio) Samples in Karacaören-2 Dam Lake. Ecology. 21 (82), 65, 2012.

2. TURCHINI G.M., TORSTENSEN B.E. Fish oil replacement in finfish nutrition. Rev. Aquacult. 1, 10, 2009.

3. WORLD HEALTH ORGANIZATION Trace elements in human nutrition and health, Genova. Number of pages: 361, Languages: English, 1996. ISBN:9241561734.http://www. who.int/nutrition/publications/micronutrients/9241561734/
4. CAHU C., SALEN P., DE LORGERIL M. Farmer and wild fish in prevention of cardiovascular diseases: assessing possible differences in lipid nutritional values. Nutrition, Metabolism \& Cardiovascular Diseases. 14, 34, 2004.

5. GANJAVI M., EZZATPANAH H., GIVIANRAD M.H., SHAMS A. Effect of canned tuna fish processing steps on lead and cadmium contents of Iranian tuna fish. Food Chem. 118, 525, 2010.

6. NEVES R.C.F., MORAES P.M., SALEH M.A.D., LOUREIRO V.R., SILVA F.A., BARROS M.M., PADILHA C.C.F., JORGE S.M.A., PADILHA P.M. FAAS determination of metal nutrients in fish feed after ultrasound extraction. Food Chemistry. 113, 679, 2009.

7. DEVIĆ G., SAKAN S., ĐORĐEVIĆ D. Assessment of the environmental significance of nutrients and heavy metal pollution in the river network of Serbia. Environmental Science and Pollution Research. 23 (1), 282, 2016.

8. KASASSI A., RAKIMBEI P., KARAGIANNIDIS A., ZABANIOTOU A., TSIOUVARAS K., NASTIS A.A. Soil contamination by heavy metals: Measurements from a closed unlined and fill. Bioresource Technology. 99, 8578, 2008 .

9. KROMHOUT D.E.B., BOSSCHIETER C.C. Lezenne The Inverse Relation Between Fish Consumption and 20-Year Mortality from Coronary Heart Disease. N Engl J Med. 312, 1205, 1985.

10. DURAL M., GOKSU M.Z., OZAK A.A. Investigation of heavy metal levels in economically important fish species captured from the Tuzla lagoon. Food Chemistry. 102, 415, 2007. doi:10.1016/j.foodchem.2006.03.001

11. TEPE Y. Metal concentrations in eight fish species from Aegean and Mediterranean Environmental Monitoring and Assessment. 159, 501, 2009.

12. ALAM M.G.M., TANAKA A., ALLINSON G., LAURENSON L.J.B., STAGNITTI F., SNOW T.E. A comparison of trace element concentrations in cultured and wild carp (Cyprinus carpio) of lake Kasumigaura, Japan. Ecotoxicology and Environmental Safety. 53, 348, 2002.

13. BOSCH A.C., NEILL B.O., SİGGE G.O., KERWATH S.E., HOFFMAN L.C. Heavy metals in marine fish meat and consumer health: a review. J. Sci. Food Agric. 96, 32, 2016.

14. GOLDEN C.D., ALLISON E.H., CHEUNG W.W., DEY M.M., HALPERN B.S., MCCAULEY D.J., SMITH M., VAITLA B., ZELLER D., MYERS S.S. Nutrition: fall in fish catch threatens human health Nature. 534, 317, 2016.

15. ZHANG L., SHI Z., ZHANG J.P., JIANG Z., WANG F., HUANG X. Spatial and seasonal characteristics of dissolved heavy metals in the east and west Guangdong coastal waters, South China. Mar. Pollut. Bull. 95, 419, 2015.

16. RAJESHKUMAR S., LIU Y., MA J., DUAN H.Y., LI $X$. Effects of exposure to multiple heavy metals on biochemical and histopathological alterations in common carp, Cyprinus carpio L. Fish. Shellfish. Immunol. 17, 1050, 2017. https://doi.org/10.1016/ j.fsi.2017.08.013.

17. LIU J.L., XU X.R., DING Z.H., PENG J.X., JIN M.H., WANG Y.S., HONG Y.G., YUE W.Z. Heavy metals in wild marine fish from South China Sea: levels, tissueand species-specific accumulation and potential risk to humans. Ecotoxicology. 24, 1583, 2015. 
18. RAJESHKUMAR S., LIU Y., ZHANG X., RAVIKUMAR B., BAI G., XIAOYU L. Studies on seasonal pollution of heavy metals in water, sediment, fish and oyster from the Meiliang Bay of Taihu Lake in China. Chemosphere. 191, 626, 2018. https://doi.org/10.1016/j. chemosphere.2017.10.078

19. GU Y.G., LIN Q., HUANG H.H., WANG L., NING J.J., DU F.Y. Heavy metals in fish tissues/stomach contents in four marine wild commercially valuable fish species from the western continental shelf of South China Sea. Marine Pollution Bulletin. 114, 1125, 2017. https://doi.org/10.1016/j. marpolbul.2016.10.040

20. VAROL M., KAYA G.K., ALP A. Heavy metal and arsenic concentrations in rainbow trout (Oncorhynchus mykiss) farmed in a dam reservoir on the Firat (Euphrates) River: Risk-based consumption advisories. Science of the Total Environment. 599, 1288, 2017. https://doi.org/10.1016/j. scitotenv.2017.05.052

21. BILGIN M., SUZER E.U. Assessment of trace metal concentrations and human health risk in clam (Tapes decussatus) and mussel (Mytilus galloprovincialis) from the Homa Lagoon (Eastern Aegean Sea). Environ Sci Pollut Res. 24, 4174, 2017. https://doi.org/10.1007/s11356-016-8163-2

22. YELTEKIN A.Ç., OĞUZ A.R. The Variations in The Levels of Some Metals in The Different Tissues of Van Fish (Alburnus Tarlchi, Guldenstadt 1814) According to Gender and Weight. Fresenius Environmental Bulletin. 26, 864, 2017.
23. QIN D., JIANG H., BAI S., TANG S., MOU Z. Determination of 28 trace elements in three farmed cyprinid fish. Food Control. 50, 1, 2015.

24. TURKISH FOOD CODEX (TFC). Regulation on contaminants in foodstuffs. Republic of Turkey Ministry of Food Agriculture and Livestock, Number: 28157, Ankara, 2011.

25. EUROPEAN COMMUNITIES COMMISSION REGULATION (EC). Setting maximum levels for certain contaminants in foodstuffs. Offcial Journal of the European Union, Commission Regulation No:1881, 2006.

26. BHOURI A.M., BOUHLEL I., CHOUBA L., HAMMAMI M., EL CAFSI M., CHAOUCH A., Total lipid content, fatty acid and mineral compositions of muscles and liver in wild and farmed sea bass (Dicentrarchus labrax). African Journal of Food Science. 4, 522, 2010.

27. AVIGLIANO E., SCHENONE N.F., VOLPEDO A.V., GOESSLER W., CIRELLI A.F. Heavy metals and trace elements in muscle of silverside (Odontesthes bonariensis) and water from different environments (Argentina): aquatic pollution and consumption effect approach. Science of the Total Environment. 506, 102, 2015.

28. ZRINKA D., NESRETE K., VLATKA F.M., DUŠICA I., MARIJANA E. Multibiomarker approach on the gills of brown trout (Salmo trutta) as a tool for the assessment of the Krka River metal pollution. Porto, Portugal: Association of Chemistry and the Environment. 26, 164, 2017. 
